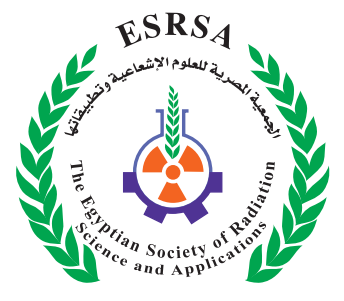

J. Nucl. Tech. Appl. Sci., Vol. 8, PP. 7 : 14 (2020)

\title{
Assessment of Diagnostic Reference Dose Levels Associated with Computed Tomography Examination
}

Taha, T.M. ${ }^{1,2}$; Alehyani, S.H. ${ }^{1}$; Mihdhir, A.A. ${ }^{1}$ and Olataiby, M. ${ }^{3}$

Received: 16/12/2019

Accepted: 04/02/2020

E.mail:tahaalfawwal@hotmail.com

\section{KEYWORDS}

Computed

Tomography,

Diagnostic Reference

Dose Levels, Volume

CT Dose index, $C T$

Air Kerma-length

Product, Effective

Dose.

\section{ABSTRACT}

The current study is aimed to estimate the diagnostic reference dose levels (DRLs), the volume computer tomogram dose index (CTDI ${ }_{\mathrm{vol}}$ ), the $\mathrm{CT}$ air kerma-length product $\left(\mathrm{P}_{\mathrm{KL}, \mathrm{CT}}\right)$ and the effective dose $(\mathrm{E})$ for head, chest and chest-abdomen-pelvic CT examinations performed on adults in King Abdullah Medical City (KAMC), Saudi Arabia. Demographic parameters (such as age, gender, weight, height) for a sample of 535 computed tomography (CT) patients and physical scan parameters (such as patient tube voltage $(\mathrm{kV})$, reference and effective mill ampere-second (mAs), scan length, number of slices, and pitch factor) were collected from a picture archiving and communication system (PACS). CTDI and $\mathrm{P}_{\mathrm{KL}, \mathrm{CT}}$ were studied for different patients' weights (40 -60 kg, 60-80 $\mathrm{kg}, 80-100 \mathrm{~kg}$ and 100-120 kg). Mean CTDI ${ }_{\mathrm{vol}}, \mathrm{P}_{\mathrm{KL}, \mathrm{CT}}$ and effective dose for a standard weight ( $70 \mathrm{~kg} \pm 10 \%)$ was evaluated according to the three $\mathrm{CT}$ protocols considered and the results were, as follows: for head examinations - $61.8 \mathrm{mGy}, 1212 \mathrm{mGy} . \mathrm{cm}$ and $2.55 \mathrm{mSv}$, respectively; for chest examinations- $13.1 \mathrm{mGy}, 657.5 \mathrm{mGy} . \mathrm{cm}$ and $9.21 \mathrm{mSv}$, respectively; for chest-abdomen-pelvic examinations - $13.9 \mathrm{mGy}, 782.5 \mathrm{mGy} . \mathrm{cm}$ and $11.73 \mathrm{mSv}$, respectively. The effective dose for the chest examinations was lower than the corresponding value communicated by the European study group under European Commission.

1. Physics Department, Faculty of Applied Sciences, Umm Al-Quraa University, KSA, P. Box 715.

2. Radiation Protection Department, Nuclear Research Center, Atomic Energy Authority, Cairo.P.O.13759 Egypt.

3. Medical Imaging Department, KAMC, Makkah City. 
The effective dose for head examinations, was slightly higher than the one communicated by the European Commission study due to differences in the scan length. It needs optimizing the radiation dose delivered to patients via periodical review of scan parameters for a protocol under study.

\section{INTRODUCTION}

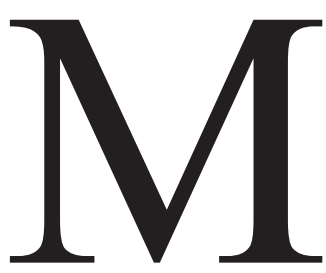

edical X-rays are the largest man-made source of public exposure to ionizing radiation. CT represented about $6 \%$ of all medical imaging procedures performed worldwide between 1997 and 2006 , and accounted for the $43 \%$ of the total dose resulting from those procedures. The contribution of CT to the collective dose in 1991-1996 was 34\% (UNSCEAR, 2010). Nowadays, the number of computed tomography [CT] examinations used in diagnostic radiology has increased in various countries of the world. Medical radiation protection principles include justification and optimization by applying the ALARA principle (as low as reasonably achievable). The DRL(s) are considered a powerful tool for optimization the concepts of diagnostic reference levels. Studies in national and international hospitals around the world have considered diagnostic reference dose levels (RDLs) for CT examinations as a basis for dose reduction (Khalid, 2016). To apply the radiation protection principle of optimization the radiation dose delivered to patients in the course of $\mathrm{CT}$ examinations, observed volume computer tomogram dose index $\left[\mathrm{CTDI}_{\mathrm{vol}}\right]$ and air kerma Length Product $\left[\mathrm{P}_{\mathrm{KL}, \mathrm{CT}}\right]$ should be compared against published diagnostic reference levels (DRLs). A lot of authors established DRL(s) in some countries around the world, such as (Suliman et al., 2011) which generated diagnostic reference dose levels for some CT examinations in Sudan. In addition to that, the American College of Radiology, (ACR, 2018) issued a new report for diagnostic reference dose levels for some CT examinations. The objectives of the current study were to study relationship between $\mathrm{CTDI}_{\text {vol }}$ and $\mathrm{P}_{\mathrm{KL}, \mathrm{CT}}$ for head, chest and chest-abdomen-pelvic examinations considering different patients weights, and to assess the dose reference levels for patients weights of $70 \mathrm{~kg} \pm 10 \%$ for the three CT dosimetric indices, $\mathrm{CTDI}_{\mathrm{vol}}$ and $\mathrm{P}_{\mathrm{KL}, \mathrm{CT}}$ and effective dose (E).

\section{MATERIAL AND METHOD}

The study was approved by King Abdullah Medical City Institution review board (IRB) no. 18-423. Two CT Siemens 64 slices with $390 \mathrm{~mm}$ ultra-fast ceramic scintillation detectors at King Abdullah Medical City scanner are used in this study. The CT Siemen 64 slices machine is presented in figure 1.

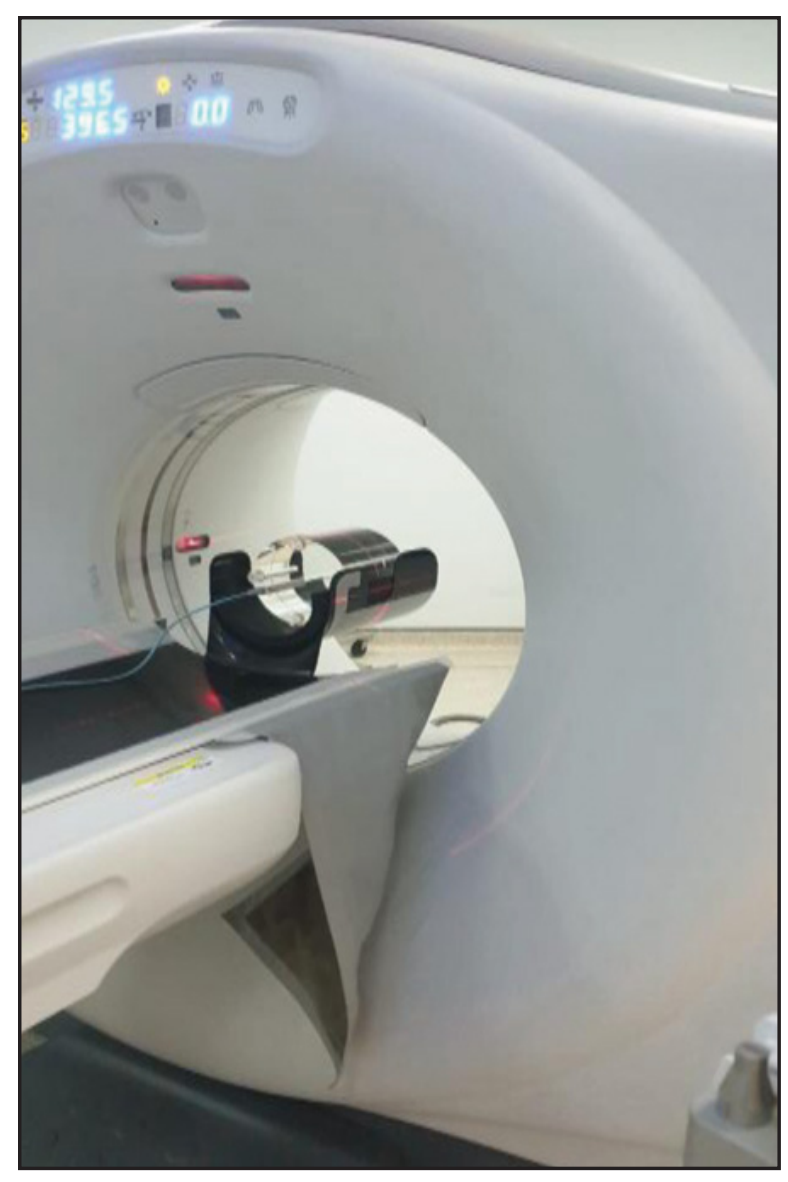

Fig. (1): GE Computed Tomography 64-MDCT. 


\section{Data Collection}

The specification of current scanner used in this study is tabulated in (table 1). Data collection of patients parameters (such as age, gender, weight, and height) and physical scan parameters (such as patient tube voltage, reference and effective mill ampere-second, section collimation, rotation time, scan length, number of slice, and pitch factor) are extracted from scanner dose report via a picture archiving and communication system (PACS) of KAMC CT scanner. Weights, heights are collected for the selected sample of patients available from the patient files. Standard dose indices, volume weighted computer tomogram dose index $\left(\mathrm{CTDI}_{\mathrm{vol}}\right)$, and CT air kerma -length product $\left(\mathrm{P}_{\mathrm{KL}, \mathrm{CT}}\right)$ for each examination were collected from picture archiving communication system. Scan parameters for the chest examination on the user interface of Siemens CT scanner are displayed on console as shown in (Fig. 2).

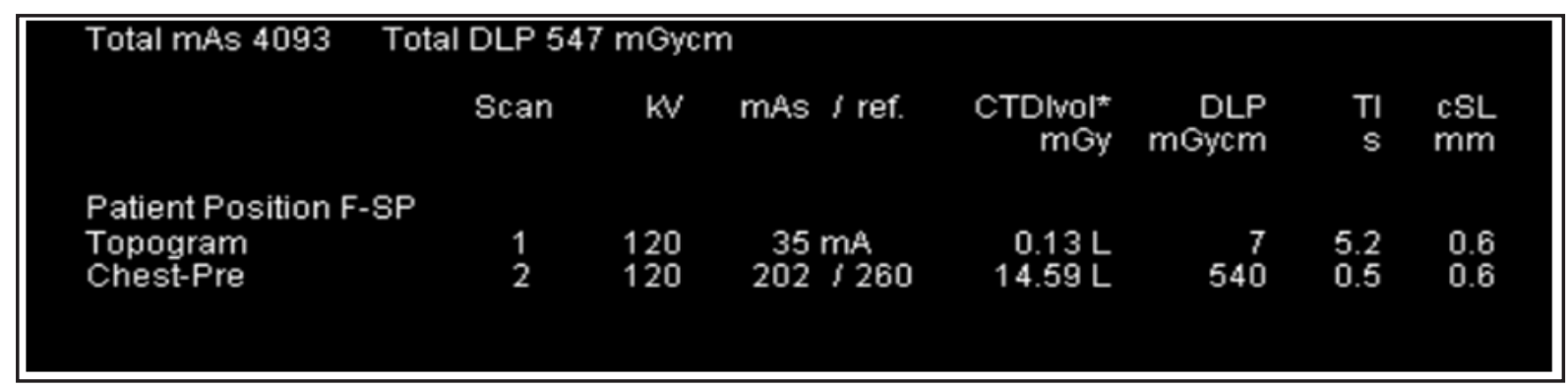

Fig. (2): Chart shows the basic dose parameters displayed on the console of a Siemens multi detector CT scanner.

\section{Quantities for CT dosimetry}

Calculation of Computer Tomography Dose Index,
CTDI $_{100}$

$\mathrm{CTDI}_{100}$ is defined as the accumulated multiple scan dose at the center of a $100 \mathrm{~mm}$ scan length in unit of mGy. The integration limits for $\mathrm{CTDI}_{100}$ are $\pm 50 \mathrm{~mm}$, which corresponds to the $100 \mathrm{~mm}$ length of the pencil ionization chamber as mentioned by International Atomic Energy Agency, (IAEA, 2014).

$\mathrm{CTDI}_{100}=\frac{1}{N T} \int_{-50}^{50} D(z) d z$

Where $\mathrm{D}(\mathrm{z})=$ the radiation dose profile along the Z-axis

$\mathrm{N}$ : The number of slice sections imaged in a single axial scan.

T: width of the slice section along the z-axis imaged by one data channel, in $\mathrm{mm}$.

\section{CT air kerma-length product $P_{K L}{ }^{\prime}{ }^{C T}$}

The CT air kerma index, $\mathrm{C}_{\mathrm{voL}}$, can be combined with patient exposure parameters to provide a dose measure for complete patient examinations. This is the $\mathrm{CT}$ air kerma-length product, $\mathrm{mGy} . \mathrm{cm}$ which is given by:

$\mathrm{P}_{\mathrm{KL}, \mathrm{CT}}=\int_{\boldsymbol{j}} \boldsymbol{n} \mathrm{C}_{\mathrm{VOL}} \mathrm{Ij}_{\mathrm{it}} \quad(\mathrm{mGy} \mathrm{cm})$

where

index $\mathrm{j}$ represents each serial scan sequence forming part of the examination;

$\mathrm{Ij}$ is the distance moved by patient couch between or during consecutive scanner rotations; and $P_{i t}$ is the total tube loading for scan sequence j. mGy/ mAs

\section{Effective Dose Calculation}

Effective dose was estimated multiplying CT air kerma-length product, $\mathrm{mGy} . \mathrm{cm}$ by a corresponding normalized conversion coefficient that is defined as specific only to the anatomic region ( $\mathrm{k}), \mathrm{mSv} / \mathrm{mGy}$. $\mathrm{cm}$ for multislice CT head, as follows: 0.0021 for 
head CT, 0.014 for chest CT, 0.015 for chest-abdomen-pelvic CT; it was stated by Sherer et al. (2018).

\section{Weighted CTDI}

The CTDI ${ }_{w}$ is a useful indicator of scanner radiation output for a specific $\mathrm{kVp}$ and $\mathrm{mAs}$. It represents the absorbed dose in two dimensions, in mGy. The weighted $\mathrm{CT}$ kerma, $\mathrm{Cw}$, combines values of $\mathrm{C}_{\mathrm{PM} \text { - }}$ $\mathrm{MA}, 100$ measured at the center and peripheral of the standard $16 \mathrm{~cm}$ head and $32 \mathrm{~cm}$ body phantoms, being given by the following relation:

$\left.\mathrm{CW}=\frac{1}{3} \mathbf{C}_{\text {PMMA,100,c }}+\frac{2}{3} \mathbf{C}_{\text {PMMA }, 100, \mathrm{p}}\right)$

The quantity $\mathrm{C}_{\mathrm{PMMA}^{\prime} 100, \mathrm{c}}$ is measured at the center of the standard CT dosimetry phantom, and $\mathrm{C}_{\mathrm{PMMA}{ }_{100 \mathrm{p}}}$ is the average of values measured at four positions around the periphery of the phantom.

\section{Volume CTDI ${ }_{\text {vol }}$}

Volume $\mathrm{CTDI}_{\mathrm{vol}}$ is defined as a volume average,
mGy, which takes account of helical pitch or axial scan spacing in three dimensions, where

$\mathrm{C}_{\mathrm{vol}}=\frac{\mathrm{Cw} \mathrm{NT}}{l}=\frac{\mathbf{C w}}{\mathbf{P}}, \mathrm{nC}_{\mathrm{vol}}=\mathrm{C}_{\mathrm{vol}} / \mathbf{P}$

1 : is defined as the distance moved by the patient couch per helical rotation or between two consecutive scans for a series of axial scans, in $\mathrm{mm}$; and $\mathrm{P}_{\mathrm{it}}$ is the tube loading for a single axial scan in $\mathrm{mGy} / \mathrm{mAs}$

The quantity $\mathrm{P}$ can be calculated by using the relation : $\mathrm{P}=\frac{l}{N T}$

$\mathrm{P}$ is known as the CT pitch factor (or pitch) for helical scanning. The quantity $\mathrm{nC}_{\mathrm{voL}}$ is unit normalized to unit tube current-exposure time product.

\section{RESULTS}

Mean \pm standard deviation of patient data and computer tomography acquisition parameters for the routine head, chest, and chest-abdomen-pelvic (body) examinations are tabulated in (table 1).

Table (1) : Patients data and main CT acquisition parameters for the considered CT protocols.

\begin{tabular}{|c|c|c|c|c|c|c|c|}
\hline \multicolumn{4}{|c|}{ Patients demographic data } & \multicolumn{4}{c|}{ Main CT acquisition parameters } \\
\hline CT protocol & $\begin{array}{c}\text { No. of } \\
\text { patients }\end{array}$ & $\begin{array}{c}\text { Age } \\
(\text { year })\end{array}$ & $\begin{array}{c}\text { Weight } \\
(\mathrm{kg})\end{array}$ & $\begin{array}{c}\text { Kilo } \\
\text { voltage } \\
\mathrm{kV}\end{array}$ & $\begin{array}{c}\text { Effective } \\
\mathrm{mAs}\end{array}$ & $\begin{array}{c}\text { Section } \\
\text { thickness } \\
(\mathrm{mm})\end{array}$ & $\begin{array}{c}\text { Number of } \\
\text { Slices }\end{array}$ \\
\hline Head & 111 & $\begin{array}{c}50 \pm \\
16\end{array}$ & $71 \pm 16$ & $120 \pm 0$ & $380 \pm 14$ & $5 \pm 3.4$ & $39 \pm 0.2$ \\
\hline Chest & 213 & $52 \pm 7$ & $\begin{array}{c}69 \pm \\
6.9\end{array}$ & $119 \pm .3$ & $236 \pm 34$ & $2.0 \pm 0$ & $94.3 \pm 10.1$ \\
\hline $\begin{array}{c}\text { Chest Abdomen- } \\
\text { Pelvic }\end{array}$ & 157 & $54 \pm 6$ & $69 \pm 7.1$ & $120 \pm 3.9$ & $196.3 \pm 34$ & $2.0 \pm 0$ & $94.3 \pm 10.1$ \\
\hline
\end{tabular}

A comparison of the dosimetric data $\mathrm{CTDI}_{\mathrm{vol}}$ and $\mathrm{P}_{\mathrm{KL}, \mathrm{CT}}$ between different patients weight intervals (such as 40-60 kg, 60-80 kg, 80-100 kg, and 100$120 \mathrm{~kg}$ ) for the head, chest, and chest- abdomen -pelvic $\mathrm{CT}$ examinations performed at the KAMC CT scanner is presented in (Table 2).
Scan data details for the 111 head CT examinations, 213 chest CT examinations and 157 chest-abdomen- pelvic CT examinations, respectively, corresponding to adult patients with a weight of $70 \mathrm{~kg}$ $\pm 10 \%$ were collected from PACS system. Standard dose indices, a volume-weighted computer tomo- 
gram dose index and CT air kerma -length product for each examination were registered via scanner report. (Table 3) presents details regarding the descriptive statistics of the surveyed examinations for dose reference levels, $\mathrm{CTDI}_{\mathrm{vol}}, \mathrm{P}_{\mathrm{KL}, \mathrm{CT}}$ and effective dose, considering the reference weight of $70 \mathrm{~kg} \pm$ $10 \%$. The current dose indices surveyed and calculated in KAMC were compared with published data by (Foley et al., 2012) including data reported by the
European Commission, (EC, 1999), Public Health UK, (UK-2011) and American College of Radiology, (ACR, 2018).

\section{Acceptance test for CTDI}

$\mathrm{CTDI}_{\mathrm{vol}}$ (mGy) for patient dosimetry is performed regularly by quality control unit of KAMC using head and body CT phantom and one sample is presented as shown in (Table 4).

Table (2) : CTDIvol ( $m G y)$ and PKL,CT ( $m G y . c m)$ considering the patients weight ranges for head, chest and chest-abdomen-pelvic examinations at KAMC, SA .

\begin{tabular}{|c|c|c|c|c|}
\hline $\begin{array}{l}\text { Dosimetric data } / C T \\
\text { examination }\end{array}$ & $40-60 \mathrm{~kg}$ & $60-80 \mathrm{~kg}$ & $80-100 \mathrm{~kg}$ & $100-120 \mathrm{~kg}$ \\
\hline \multicolumn{5}{|c|}{$\mathrm{C}_{\mathrm{vol}}(\mathrm{mGy})$} \\
\hline $\begin{array}{c}\text { Head } \\
\text { Chest } \\
\text { Abdomen-Pelvic- Chest }\end{array}$ & $\begin{array}{l}61 \pm 6.0 \\
9 \pm 2.5 \\
11 \pm 2.7\end{array}$ & $\begin{array}{l}62 \pm 2.0 \\
13 \pm 4.5 \\
14 \pm 3.5\end{array}$ & $\begin{array}{c}57 \pm 14 \\
17 \pm 4.0 \\
19 \pm 4.0\end{array}$ & $\begin{array}{l}59 \pm 10 \\
20 \pm 3.0 \\
22 \pm 3.0\end{array}$ \\
\hline \multicolumn{5}{|c|}{$P_{\mathrm{KL}, \mathrm{CT}}(\mathrm{mGy} \cdot \mathrm{cm})$} \\
\hline $\begin{array}{c}\text { Head } \\
\text { Chest } \\
\text { Chest-Abdomen }- \text { Pelvic }\end{array}$ & $\begin{array}{l}1197 \pm 148 \\
490 \pm 214 \\
659 \pm 243\end{array}$ & $\begin{array}{l}1212 \pm 67 \\
658 \pm 278 \\
783 \pm 238\end{array}$ & $\begin{array}{c}1144 \pm 258 \\
810 \pm 353 \\
1042 \pm 289\end{array}$ & $\begin{array}{r}1278 \pm 462 \\
1008 \pm 399 \\
1331 \pm 319\end{array}$ \\
\hline
\end{tabular}

Table (3) : CTDIvol ( $m G y)$ and PKL,CT ( $m G y . c m)$ considering the patients weight ranges for head, chest and chest-abdomen-pelvic examinations at KAMC, SA .

\begin{tabular}{|c|c|c|c|c|}
\hline $\begin{array}{l}\text { Dosimetric data/ } \\
\text { CT examination }\end{array}$ & KAMC & UK-2011 & $E C, 1999$ & ACR-2018 \\
\hline \multicolumn{5}{|c|}{$\mathrm{C}_{\mathrm{vol}}(\mathrm{mGy})$} \\
\hline Head & $61.8 \pm 2.4$ & 61 & 60 & 56 \\
\hline Chest & $13.1 \pm 4.5$ & 12 & 30 & 14 \\
\hline Chest-Abdomen-Pelvic & $13.9 \pm 3.5$ & 12 & - & 15 \\
\hline \multicolumn{5}{|c|}{$P_{\mathrm{KL}, \mathrm{CT}} \quad(\mathrm{mGy} . \mathrm{cm})$} \\
\hline Head & $1212 \pm 67$ & 970 & 1050 & 962 \\
\hline Chest & $657 \pm 4.5$ & 610 & 650 & 469 \\
\hline Chest-Abdomen -Pelvic & $783 \pm 238$ & 1000 & - & 947 \\
\hline \multicolumn{5}{|c|}{ E (mSv) } \\
\hline Head & $2.55 \pm 0.14$ & - & 2.4 & - \\
\hline Chest & $9.21 \pm 0.06$ & - & 11.7 & - \\
\hline Chest-Abdomen-Pelvic & $11.73 \pm 3.56$ & - & - & - \\
\hline
\end{tabular}


Table (3) : CTDIvol ( $m G y)$ and PKL,CT ( $m G y . c m)$ considering the patients weight ranges for head, chest and chest-abdomen-pelvic examinations at KAMC, SA .

\begin{tabular}{|c|c|c|}
\hline Examination & $\begin{array}{c}\text { Pass /fail criteria } \\
\text { CTvol (mGy) }\end{array}$ & $\begin{array}{c}\text { Reference levels } \\
\text { CTDIvol (mGy) (ACR,2008) }\end{array}$ \\
\hline Adult head & 60 & 75 \\
\hline Adult Abdomen & 22 & 25 \\
\hline
\end{tabular}

The relative percentage error between calculated and reference levels of Cvol of head and abdomen are lower than those reported by American College of Radiology, (ACR,2018) as mentioned in table

\section{DISCUSSION}

The CTDI ${ }_{\mathrm{vol}}$ and $\mathrm{P}_{\mathrm{KL}, \mathrm{CT}}$ corresponding to three CT protocols (head, chest and chest-abdomen-pelvis examinations) were collected from a picture archiving and communication system (PACS) for patients weight ranges 40-60 kg, 60- $80 \mathrm{~kg}, 80-100 \mathrm{~kg}$ and $100-120 \mathrm{~kg}$, respectively. As it can be seen in (table.2), CTDI ${ }_{\text {vol }}$ corresponding to the head CT examinations for different weight ranges were found to be $60.5 \pm 6.1 \mathrm{mGy}(40-60 \mathrm{~kg}), 61.7 \pm 2.3 \mathrm{mGy}$ $(60-80 \mathrm{~kg}), 57 \pm 14 \mathrm{mGy}(80-100 \mathrm{~kg})$, and $59.4 \pm$ 10.3 mGy (100-120 kg), respectively. $\mathrm{P}_{\mathrm{KL}, \mathrm{CT}}$ values corresponding to the head CT examinations for the considered weight ranges were found to be $196.7 \pm$ 147.6 mGy.cm (40-60 kg ), $1212 \pm 67$ mGy.cm (60$80 \mathrm{~kg}), 1144 \pm 258 \mathrm{mGy} . \mathrm{cm}(80-100 \mathrm{~kg})$, and 1278 $\pm 462.22 \mathrm{mGy} . \mathrm{cm}(100-120 \mathrm{~kg})$, respectively. There is no significant change in $\mathrm{CTDI}_{\mathrm{vol}}$ and $\mathrm{P}_{\mathrm{KL}, \mathrm{CT}}$ for the head CT protocol that can be explained by the number of slices and scan length approximately constant.

CTDI $_{\text {vol }}$ characterizing the chest CT examinations for different patients weights were found to be $9.3 \pm 2.5$ mGy (40-60 kg), $13.6 \pm 7$ mGy (60$80 \mathrm{~kg}), 16.5 \pm 6.7 \mathrm{mGy}(80-100 \mathrm{~kg})$, and $20.4 \pm 3$ (100-120 kg), respectively (see table.2). $\mathrm{P}_{\mathrm{KL}, \mathrm{CT}}$ corresponding to the chest $\mathrm{CT}$ examinations for different weight ranges were found to be $489.7 \pm 214 \mathrm{mGy} . \mathrm{cm}$ (40-60 kg), $658 \pm 278$ mGy.cm $(60-80 \mathrm{~kg}), 810 \pm$ 353 mGy.cm (80-100 kg), and 1007.6 $\pm 399.4 \mathrm{mGy}$. cm (100-120 kg), respectively. $\mathrm{CT}_{\mathrm{vol}}$ represents the average absorbed radiation dose over the $\mathrm{x}, \mathrm{y}$, and z-direction, whereas $\mathrm{P}_{\mathrm{KL}, \mathrm{CT}}$ represents the integration of absorbed dose along the scan length of head patient's sizes.

CTDI $_{\mathrm{vol}}$ corresponding to the chest-abdomenpelvic $\mathrm{CT}$ examinations for the considered patients weight ranges were found to be $11 \pm 2.7 \mathrm{mGy}$ (40-60 $\mathrm{kg}), 14 \pm 3.5 \mathrm{mGy}(60-80 \mathrm{~kg}), 19 \pm 4.0 \mathrm{mGy}(80-$ $100 \mathrm{~kg})$, and $22 \pm 3 \mathrm{mGy}(100-120 \mathrm{~kg})$, respectively. $\mathrm{P}_{\mathrm{KL}, \mathrm{CT}}$ corresponding to the chest-abdomen-pelvic $\mathrm{CT}$ examinations for different patients weight ranges were found to be $659 \pm 243 \mathrm{mGy} . \mathrm{cm}(40-60 \mathrm{~kg})$, $783 \pm 238$ mGy.cm (60-80 kg), $1042 \pm 289$ mGy. $\mathrm{cm}(80-100 \mathrm{~kg})$, and $1331 \pm 319$ mGy.cm (100-120 $\mathrm{kg}$ ), respectively. $\mathrm{CTDI}_{\mathrm{vol}}$ f11 or chest and chest-abdomen-pelvic $\mathrm{CT}$ examinations for different patients weight ranges increased gradually due to increasing of the number of slices, the slice thickness, and the pitch factor in addition to decreasing of the effective milli amper sec. $\mathrm{P}_{\mathrm{KL}, \mathrm{CT}}$ for chest and chest-abdomenpelvic CT examinations for different patients weight ranges increased gradually due to increasing of the number of slices, the slice thickness, and the scan length.

$\mathrm{CTDI}_{\mathrm{vol}}$ for CT reference patient weight $(70 \mathrm{~kg}$ $\pm 10 \%$ ) corresponding to the head CT examinations 
showed the smallest variation (see table.3) comparatively with the values reported by the considered foreign studies due to the non-significance difference in the scan length of the patients head size. CTDIvol for the chest CT examinations was found to be close to those reported by the Public Health England (UK, 2011) and the American College of Radiology (ACR, 2018), and 50\% lower than that reported by the European Commission study (Bongartz et al., 2004) due to the short scan length. $\mathrm{CTDI}_{\mathrm{vol}}$ for the chest-abdomen-pelvic CT examinations was found to be close to that reported by Public Health England (UK, 2011) and lower than the one reported by the American College of Radiology (ACR, 2018).

$\mathrm{P}_{\mathrm{KL}, \mathrm{CT}}$ for chest and chest-abdomen-pelvic CT examinations for different patients weight ranges increased gradually due to increasing of the number of slices, the slice thickness, and the scan length. $\mathrm{P}_{\mathrm{KL}, \mathrm{CT}}$ for head CT was higher than the dose found by European committee $(\mathrm{EC}, 1999)$ by $3 \%$. $\mathrm{P}_{\mathrm{KL}, \mathrm{CT}}$ for the chest CT examinations was found to be little higher than values reported by the Public Health England (UK, 2011) and the American College of Radiology (ACR, 2018), and $7.7 \%$ higher that reported by the European Commission study (Bongartz et al., 2004) due to the little increment scan length. $\mathrm{P}_{\mathrm{KL}, \mathrm{CT}}$ for the chest-abdomen-pelvic CT examinations was found to be close to that reported by the American College of Radiology (ACR, 2018) and lower than the one reported by Public Health England (UK, 2011) by $20.7 \%$ due to difference in scan length.

The effective dose for the head CT examinations was slightly higher than the European Commission study due to differences in the scan length. The scan length of the KAMC head CT protocol was found higher than the value of $144 \mathrm{~mm}$ presented by Suleiman et al. (2011) and the value of $150 \mathrm{~mm}$ presented by ACR (2018). The effective dose for a reference patient weight of $70 \mathrm{~kg} \pm 10 \%$ in the chest CT protocol case was about $20 \%$ lower than the one reported by the European committee as presented in table 3 due to the short scan length and using of a new advanced software, CARE Dose $4 \mathrm{D}$ for $\mathrm{CT}$ imaging. Table 4 presented the effective dose of chest-abdomen-pelvic was found to be $11.7 \mathrm{mSv}$ close to the one reported by the (UK, 2006).

\section{CONCLUSION}

Calibration of all dosimeters used for patient dosimetry should be performed regularly and should be traceable to a primary standard laboratory. There is no significant change in CTDI ${ }_{\mathrm{vol}}$ and $\mathrm{P}_{\mathrm{KL}, \mathrm{CT}}$ for the head because of the number of slices and scan length approximately constant. $\mathrm{CTDI}_{\mathrm{Vol}}$ for chest and chest-abdomen-pelvic CT examinations for different patient's weights increased gradually due to increasing number of slices and slice thickness and the pitch factor. $\mathrm{P}_{\mathrm{KL}, \mathrm{CT}}$ for chest and chest-abdomen-pelvic CT examinations for different patient's weights increased due to increasing in scan length. Diagnostic Reference dose levels for patients weights of $70 \mathrm{~kg}$ $\pm 10 \%$ were established. The effective dose for the chest was lower than the corresponding value of European Commission study due to more advanced CT technology used at KAMC. The effective dose for head examinations was slightly higher than the European Commission study due to use approximately similar scan length. Local DRLs and typical values may be used as additional tools for optimization by periodical review of scan parameters for a protocol under study.

\section{ACKNOWLEDGMENTS}

This work was supported by Radiology Department of King Abdullah Medical City, KAMC, Makkah, KSA. 


\section{REFERENCES}

- American College of Radiology, ACR. (2018): General Diagnostic Radiology Practice parameter, Available at_www.acr.org.

- International Atomic Energy Agency, IAEA. (2014): Dosimetry in Diagnostic Radiology: An International Code Practice, Technical Reports Series no. 457 , IAEA.

- Bongartz, G.; Golding, S.J.; Jurik, A.G.; Leonardi, M.; van Persijn van Meerten, E.; Rodríguez, R.; Schneider, K.; Calzado, A.; Geleijns, J.; Jessen, K.A.; Panzer, W.; Shrimpton, P.C. and Tosi, G. (2004): European guidelines for multislice Computed tomography, European Commission.

- Khalid, A. (2016): Radiation Protection in X-ray Computed Tomography, Literature Review. Int. J. Radiol. Imaging. Technol., 2:016.

- Sherer, M.A.S.; Visconti, P.J.; Russell, R.E. and Haynes, K.W. (2018): Radiation Protection in Medical Radiography, 8th Edition, E book, Elsevier.
- Shrimpton, P.C.; Hillwer, M.C.; Lewis, M.A. and Dunn, M. (2006): National survey of doses from CT in the UK:2003. Br. J. Radiol., 79(948): 968.

- Foley, S.J.; Mcentee, M.F. and Rainford, L.A. (2012): Establishment of CT diagnostic reference levels in Ireland. Br. J. Radiol., 85(1080):1390.

- Shrimpton, P.C.; Hiller, M.C.; Meeson, S. and Golding, S.J. (2014): Doses from computed tomography (CT) Examination in the UK-2011 Review. PHE-CRCE-013Public Health England.

- Suliman, I.I.; Abdalla, S.E.; Nada, A.A.; Ahmed, M.A. and Galal, I.A. (2011): Survey of Computed tomography technique and radiation dose in Sudanese hospitals. Eur. J. Radiol. 80(3): e544.

- United Nations Scientific Committee on Effects of Radiation Atomic Radiation (UNSCEAR), (2000): Sources and effects of ionizing radiation report to the general assembly. New York: United Nations. 